\title{
CHARACTERIZING AND TESTING A FABRIC CONTAINING AMORPHOUS GLASS-COATED FERROMAGNETIC MICROWIRES FOR ITS MAGNETIC AND ELECTRIC SHIELDING PROPERTIES IN THE FREQUENCY RANGE (1-3) GHZ
}

Simona MICLĂUŞ*

simo.miclaus@gmail.com

Lucian BARBU-TUDORAN**

lucianbarbu@yahoo.com

Paul BECHET*

pbechet@gmail.com

Octavian BALTAG***

octavian.baltag@bioinginerie.ro

* “NICOLAE BÄLCESCU” LAND FORCES ACADEMY, SIBIU, ROMANIA

**NATIONAL INSTITUTE FOR R\&D OF ISOTOPIC AND MOLECULAR TECHNOLOGIES, CLUJ-NAPOCA, ROMANIA

***TERRAFLUX CONTROL, IAŞI, ROMANIA

\begin{abstract}
By applying a non-standardized methodology and by using electric- and magnetic-field probes of small dimensions $(<1 \mathrm{~cm})$, we experimentally characterized the electromagnetic shielding properties of a fabric containing ferromagnetic microwires weaved on a single direction. Electronic microscopy and $X$-ray spectroscopy revealed the structure, dimensions and chemical elements content of the amorphous magnetic material. Electric shielding factor proved to be very low in the investigated frequency range, but magnetic shielding factor was high, especially when the weaving direction of the magnetic wires corresponded to the polarization direction of the emitting antenna, and showed some resonances. The magnetic shielding efficiency, if checked against an increasing incident magnetic flux density, proved not to change up to $200 \mathrm{nT}$. The investigated fabric have been previously proved to be very efficient in shielding the field emitted by a mobile phone in its near field, but present results show that near and far field shielding properties are different.
\end{abstract}

\section{KEYWORDS:}

Electromagnetic shielding, amorphous ferromagnetic wires, near field probe, magneto-impedance effect, amorphous material microscopy 


\section{Introduction}

A category of composite materials with very interesting features related to electric and especially the magnetic shielding properties at microwave frequencies is the one which contains amorphous glass-coated ferromagnetic wires (Makhnovskiy \& Panina, 2006; Yong-jiang, et al., 2007; Liberal, 2013; Devkota et al., 2014; Baranov, Larin, \& Torcunov, 2017). The amorphous wire consists of a metallic core made of a complex alloy which is coated by glass, generally Pyrex. The core has typically a diameter in the order of microns, while the main alloy metallic elements are usually $\mathrm{Co}, \mathrm{Fe}, \mathrm{Ni}, \mathrm{Mn}$. The presence of $\mathrm{Si}$, $\mathrm{B}, \mathrm{C}, \mathrm{Mo}$ in the envelope (with a thickness of few microns) provides specific amorphicity to the alloy. Besides improved mechanical properties of amorphous than the crystalline counterparts of the elements (due to lack of long-range chemical and topological order) and good electric properties (however 100 times smaller electric conductivities than the crystalline counterparts), the amorphous ferromagnetic wires present exciting magnetic properties especially at high frequencies (microwaves), such as large Barkhausen jump, giant magneto-impedance and natural ferromagnetic resonance (Liberal, 2013). The magnetic properties are a function of internal composition and geometry, being dictated by the dimensions of the wires and by the sign and absolute value of the magnetostriction constant. Due to the special magnetic properties, amorphous ferromagnetic wires have diverse applications: reconfigurable devices, contactless sensors, electromagnetic absorbers and metamaterials.

Present experimental approach aimed to characterize a sheet of amorphous fabric for its electromagnetic shielding properties, by first analyzing its composition and internal geometry by means of electronic microscopy and then by preliminary testing the shielding efficiency, separately for the electric (E) and for the magnetic $(\mathrm{H})$ field components of an electromagnetic wave travelling through it. The motivation of present study is based on some previous findings of ours (Miclaus, et al., 2017) which showed that this fabric used to cover a mobile phone which emitted at $0.89 \mathrm{MHz}$ and at $1.95 \mathrm{GHz}$, greatly diminished (with $70-76 \%$ ) the specific absorption rate (SAR) of electromagnetic energy deposited inside a phantom head wearing the covered terminal at the ear. Therefore we considered important to investigate this time a larger frequency range, (1-3) $\mathrm{GHz}$, and in conditions of far-field irradiation (but applying a non-standardized protocol) and to identify which component of the wave is shielded more, $E$ or $H$. In the case of previous SAR measurements, the probe used in the standardized protocol was an E-field one, which was immersed in the liquid filling the phantom head exposed to radiation from the mobile phone, and the measurement took place in the near field of the emitting antenna.

The objectives of the present experiments were two:

a) assessing the shielding efficiency of the fabric in function of frequency, at the same incident field strength;

b) assessing the attenuation factor change (if any) when the magnitude of the incident $\mathrm{H}$-field strength is modified, preserving the frequency.

\section{Materials and methods}

The amorphous specimen of the fabric was analyzed with a field-emission-gun scanning electron microscope (SEM), $30 \mathrm{kV}$, with secondary electron imaging capability model Hitachi SU8230 (Japan). Energydispersive X-ray spectroscopy (EDS) (Oxford Instruments, UK) was then used for chemical elements identification in the sample.

The electromagnetic shielding properties were determined using a non-standardized set-up and methodology, based on field probes (for E- and $\mathrm{H}$ - field) usually used for near-field measurements (of small dimensions / few millimeters). By placing each one in the direction of maximum gain of the directive emitting antenna, and at the limit distance separating the near - and the far- 
field zone, we were able to record the very local field strength levels in the absence and then in the presence of the fabric sheet (this one was interposed between emitting antenna and field probe). By making the difference, the shielding efficiency or, similar, the attenuation factor expressed in $\mathrm{dB}$ was calculated. It was not necessary to make the experiments in free space or in an anechoic room because:

a) from the frequency $1 \mathrm{GHz}$ upward, the wavelengths are smaller than $30 \mathrm{~cm}$, and therefore the influence of the walls and nearby objects may be neglected;

b) since the field probes are smaller than $1 \mathrm{~cm}$, the effective area of the receiving sensor is also small and so the expressed field level is very localized in space. Each measurement was repeated three times and the deviation from the mean was always less than $1.14 \mathrm{~dB}$.

The emission system was composed of a log periodic broadband Yagi antenna model HyperLOG 7060 (Aaronia) and a SMBV vector signal generator (Rohde $\&$ Schwarz), providing an input power of $20 \mathrm{dBm}$ (Figure no. 1a). The receiving system consisted of a FSVR real time spectrum analyzer (Rohde \& Schwarz) connected sequentially to a $\mathrm{H}$-field probe model PBS1-H1 (Aaronia) and an E-field probe model PBS1-E1 (Aaronia). The real

a)

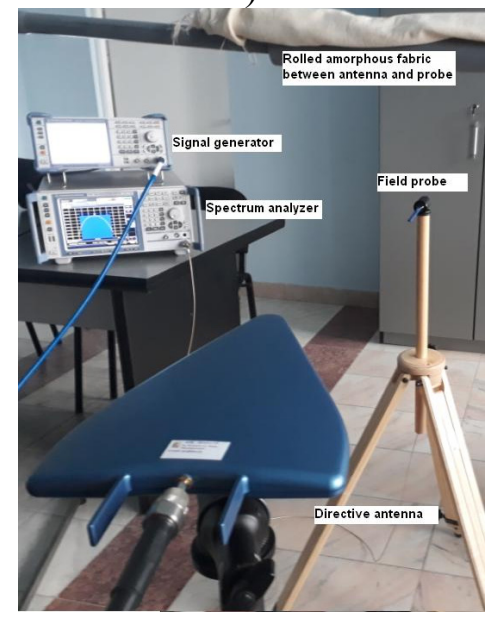

time spectrum analyzer settings were: resolution bandwidth $=3 \mathrm{kHz}$; video bandwidth $=30 \mathrm{kHz}$; the emitted signal was captured in the channel power mode, with a channel bandwidth of $10 \mathrm{kHz}$. The very local measurement capability of the probes together with the high azimutal directivity of the hyperlog antenna (Figure no. 1b) enables the reduction of the usable surface of the shielding material needed for the experimental testing $(60 \times 60 \mathrm{~cm})$. The height above the ground at which the antenna and the field probes were placed was of $107 \mathrm{~cm}$, while the horizontal distance between the antenna tip and the probe tip was of $50 \mathrm{~cm}$.

The fabric was placed vertically in two positions:

a) at half distance between the field probe and the antenna $(25 / 25 \mathrm{~cm})$;

b) nearer to the probe $(10 / 40 \mathrm{~cm})$.

These two situations were analyzed, in order to observe a possible significant influence of the position. Since the fabric contains magnetic wires on one single direction, we used it in two orientations, rotated with 90 degrees from one to another:

a) when the E-field was received parallel to the magnetic wires;

b) when the E-field was received perpendicular to them.

b)

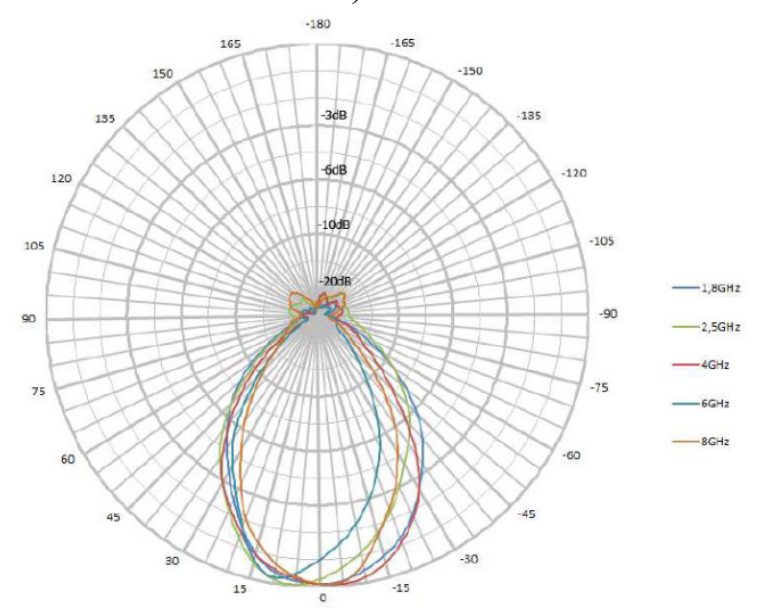

Figure no. 1 a) The experimental set-up used for preliminary testing of the magnetic and electric shielding capabilities of the amorphous glass-coated ferromagnetic microwires fabric; b) Horizontal pattern of the Hyperlog 7060 antenna used at emission (provided by the producer, Aaronia A.G.). 
The fabric used in the current measurements has been previously analyzed by (Baltag et al., 2000; Rau, Iftemie, Baltag, \& Costandache, 2011). The magnetic properties of the wires are influenced by the core-alloy elements as well as by the distribution of internal stresses (Liberal, 2013). Fe-rich wires have a completely different magnetic behavior then Co-rich wires. The distribution of the internal stresses induced during the fabrication process of such materials depends on the dimensions of the wires. Another significant parameter is the ratio between the inner core diameter and the total wire diameter. While any change in the internal stress modifies the magnetic behavior, one has to take into account that in the present experiment the fabric have previously suffered a mechanical wash, fact that might have been affected the coating integrity, with some properties change consequences.

Since magneto impedance effect is present in amorphous ferromagnetic materials in the $\mathrm{MHz}-\mathrm{GHz}$ frequency ranges, and also ferromagnetic resonance and good microwaves absorption properties are expected, a second objective of the approach was to follow the attenuation factor variation in function of the incident field strength, at the same frequency. In this regard, we used a radiofrequency power amplifier Ophir 5150 connected to the
SMBV vector signal generator, to enable incident field magnitude modification (input power into the antenna could be increased up to $33 \mathrm{~W}$ ).

\section{Results and discussion}

Figure no. 2 presents the scanning electron micrographs of the fabric: glasscoated amorphous ferromagnetic microwires are twisted together with cotton/synthetic yarns. The dimensions and geometry are clearly observable: there exist two main yarning directions, however sparse wires are present also (the mechanical washing effect). The ferromagnetic wires are however present on a single direction. The largest wires have an external diameter of $22 \mu \mathrm{m}$ while the smallest ones have diameters of $12 \mu \mathrm{m}$.

In Figure no. 3 a detailed image of an amorphous wire is shown; the elemental analysis of its core reveals the presence of $\mathrm{Co}, \mathrm{Fe}, \mathrm{Ni}, \mathrm{Mn}, \mathrm{B}$ and $\mathrm{Si}$. Other trace elements revealed by the analysis were: $\mathrm{Ti}$, $\mathrm{Hf}, \mathrm{Ca}, \mathrm{Na}, \mathrm{K}, \mathrm{Cl}, \mathrm{N}$ and of course $\mathrm{C}$ and $\mathrm{O}$. The diameter of the amorphous wire core is between (10-13) $\mu \mathrm{m}$ and the Pyrex coat is between (3-4) $\mu \mathrm{m}$ thick.

Figure no. 4 reveals the spectroscopic images in X-ray of the chemical elements distribution over the fabric and the intimate positions of some of the elements.

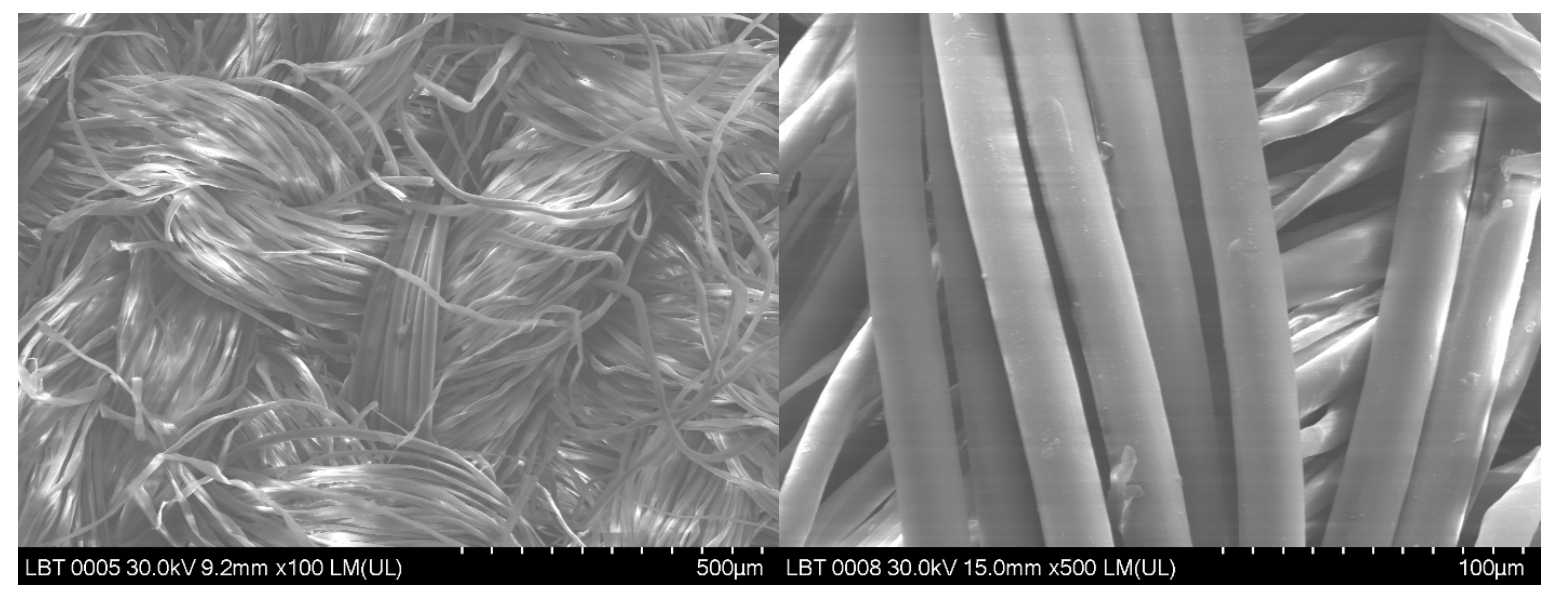

Figure no. 2 Scanning electron micrograph of the amorphous glass-coated ferromagnetic microwires - which are the bigger diameter and smooth surface's ones - among the fabric (cotton) wires 

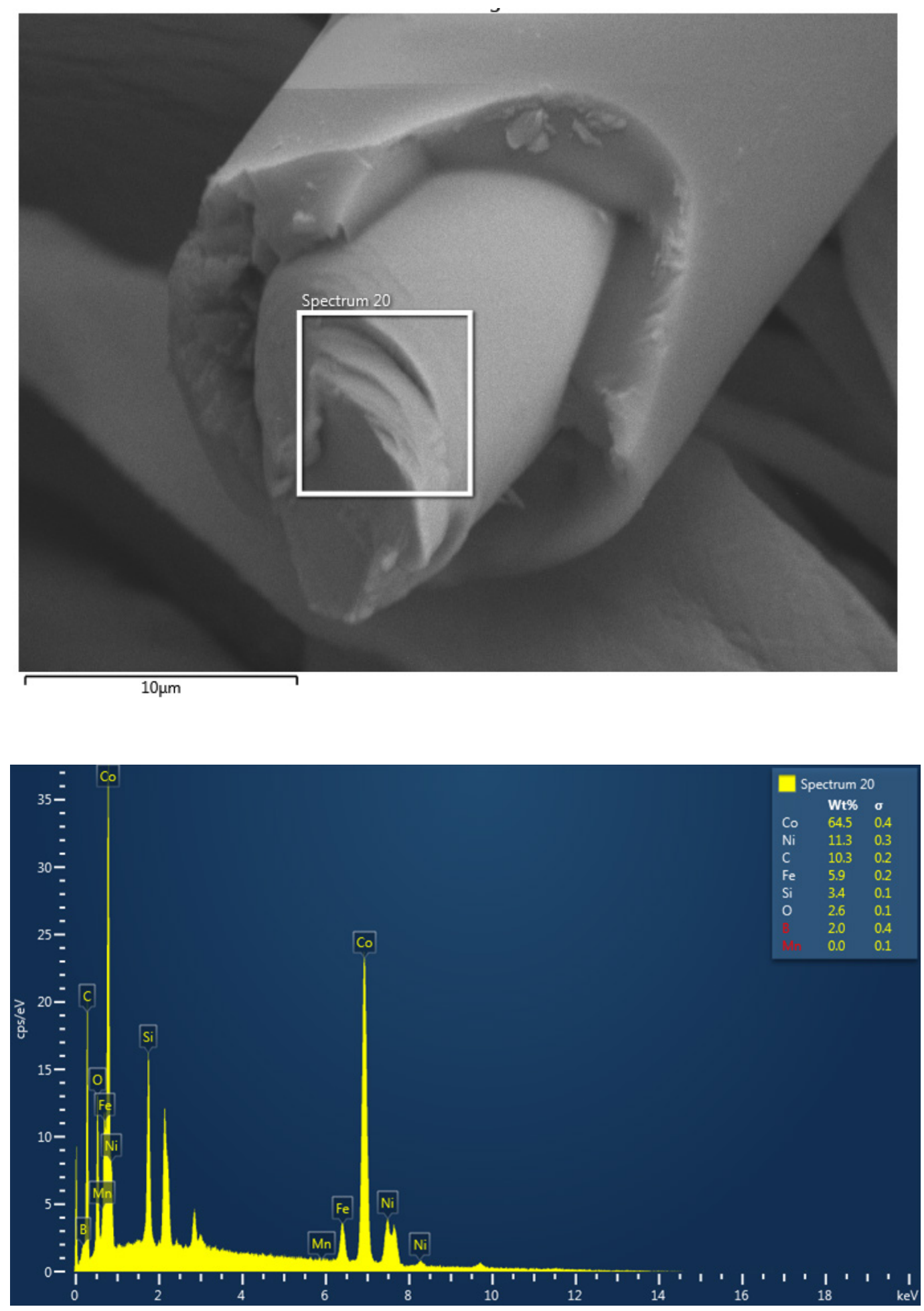

Figure no. 3 Scanning electron microscopy and elemental analysis.

Scanning electron image produced with secondary electrons accelerated at $30 \mathrm{kV}$ on a fractured wire and EDS analysis showing strong peaks for $\mathrm{Co}, \mathrm{Si}, \mathrm{Fe}, \mathrm{Ni}$ 


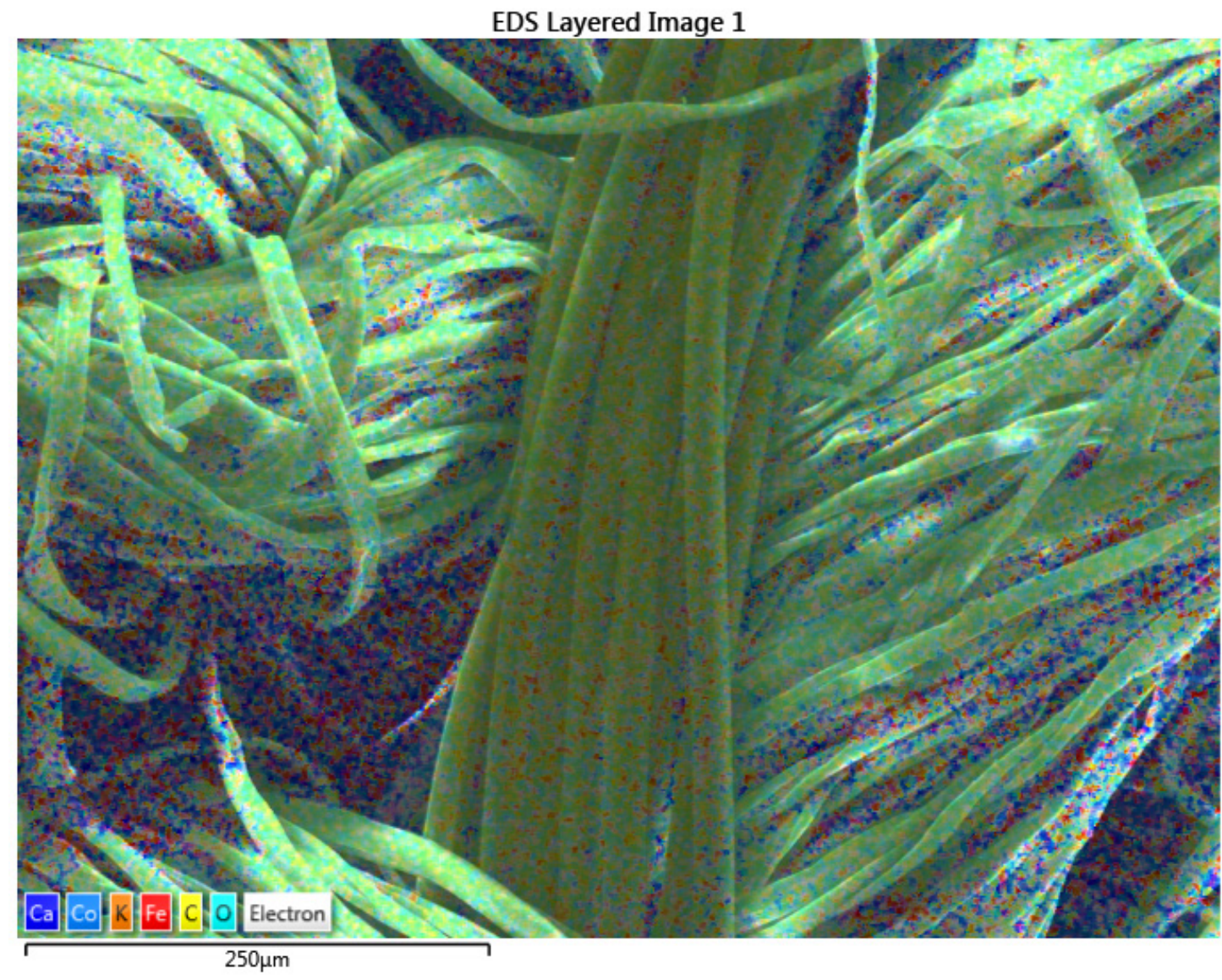

tile

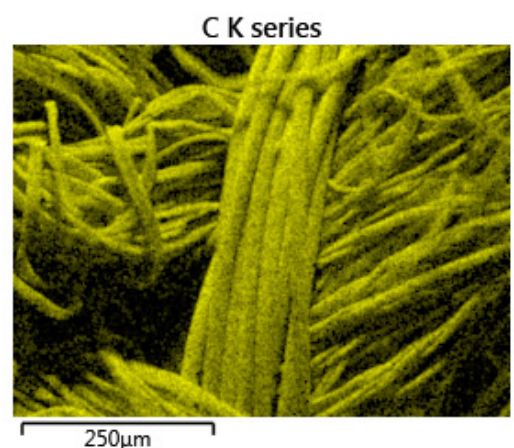

$\mathrm{O} \mathrm{K}$ series

K K series
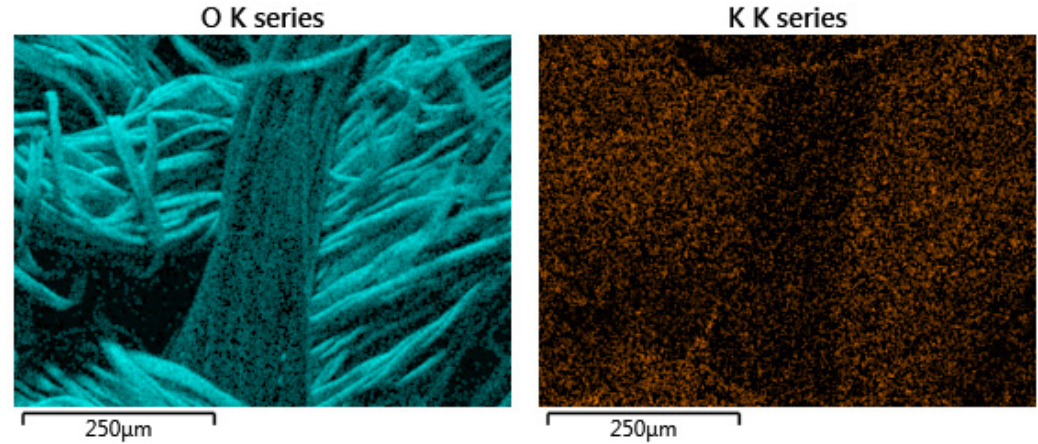

Fe K series
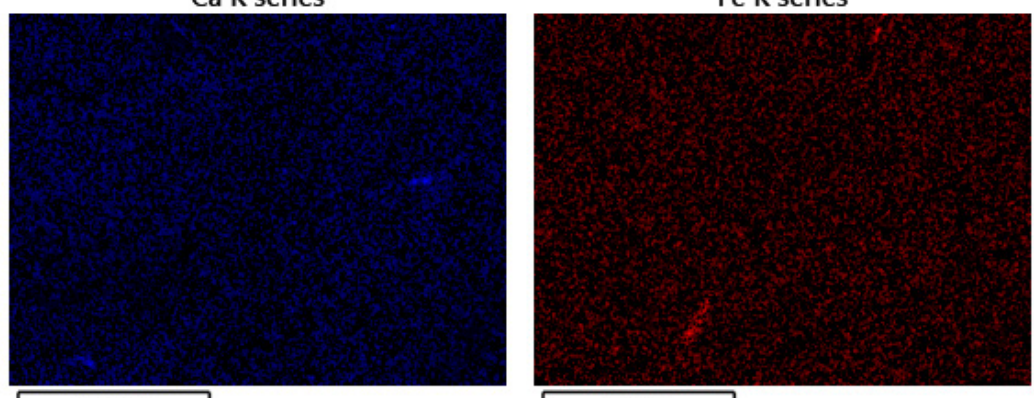

Co K series

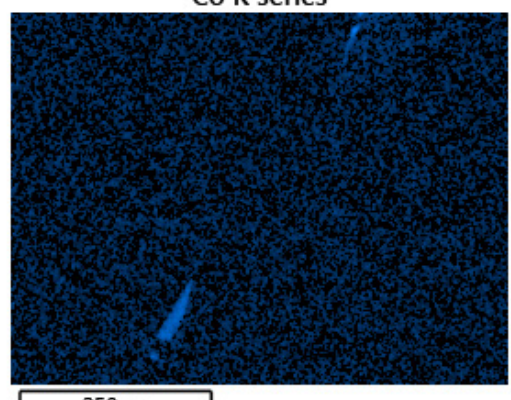

$250 \mu \mathrm{m}$

Figure no. 4 EDS mapping showing the elements distribution in the amorphous wires and in the textile 
Electromagnetic sounding of this fabric specimen revealed the consistent attenuation of the $\mathrm{H}$-field component of the wave in the whole investigated frequency range, with resonances at some frequencies. Fig. 5 shows the variation of the $\mathrm{H}$ - and E-field strength attenuation with frequency, in two situations: when the ferromagnetic wires were parallel to the horizontal polarization of the antenna, i.e. wires were parallel to E-field lines, and when they were perpendicular to them. Since E-field component attenuation was small in both orientations of the fabric, only one curve is exemplified in Figure no. 5 (when the shield was positioned at the half distance between antenna and field probe, and with the amorphous wires parallel to E-field lines). E-field is attenuated significantly $(>5 \mathrm{~dB}$ ) only for a few very narrow bandwidths around $1.1 \mathrm{GHz}, 2.6 \mathrm{GHz}$ and $2.9 \mathrm{GHz}$. Significant attenuation suffers the $\mathrm{H}$-field component over the entire range between (1-3) $\mathrm{GHz}$, but only when the ferromagnetic wires are parallel to E-field vector (red and blue curves). The differences between red and blue curves are due to positioning the fabric nearer to the probe (red). One can observe that starting from
$2.1 \mathrm{GHz}$ upward, practically the position of the shield relative to the probe has no more impact, fact that is connected with an enough decrease of the wavelength so that various wave propagation phenomena (diffraction, multiple reflexions, etc.) to not count much in the result. Generally, from $1.4 \mathrm{GHz}$ upward, a shielding efficiency of $10-25 \mathrm{~dB}$ was obtained for the $\mathrm{H}$-field component.

Comparing present results to the older ones referring to SAR values decrease due to using the fabric to cover the mobile phone (Miclaus, et al., 2017), one can address only one frequency - of $1.95 \mathrm{GHz}$. Present measurements show that only the $\mathrm{H}$-field component is shielded by the fabric, and that shielding efficiency of $\mathrm{H}$-field level at this frequency is in the range (14-17) dB. The SAR reduction factor at the same frequency was 0.47 and 0.69 respectively, depending on the amorphous wires direction. Since SAR is proportional to squared E-field strength, it results that SAR reduction was 5-6 times smaller than the equivalent field strength reduction obtained in the present experiment. This difference may be due farto-near-field differences.

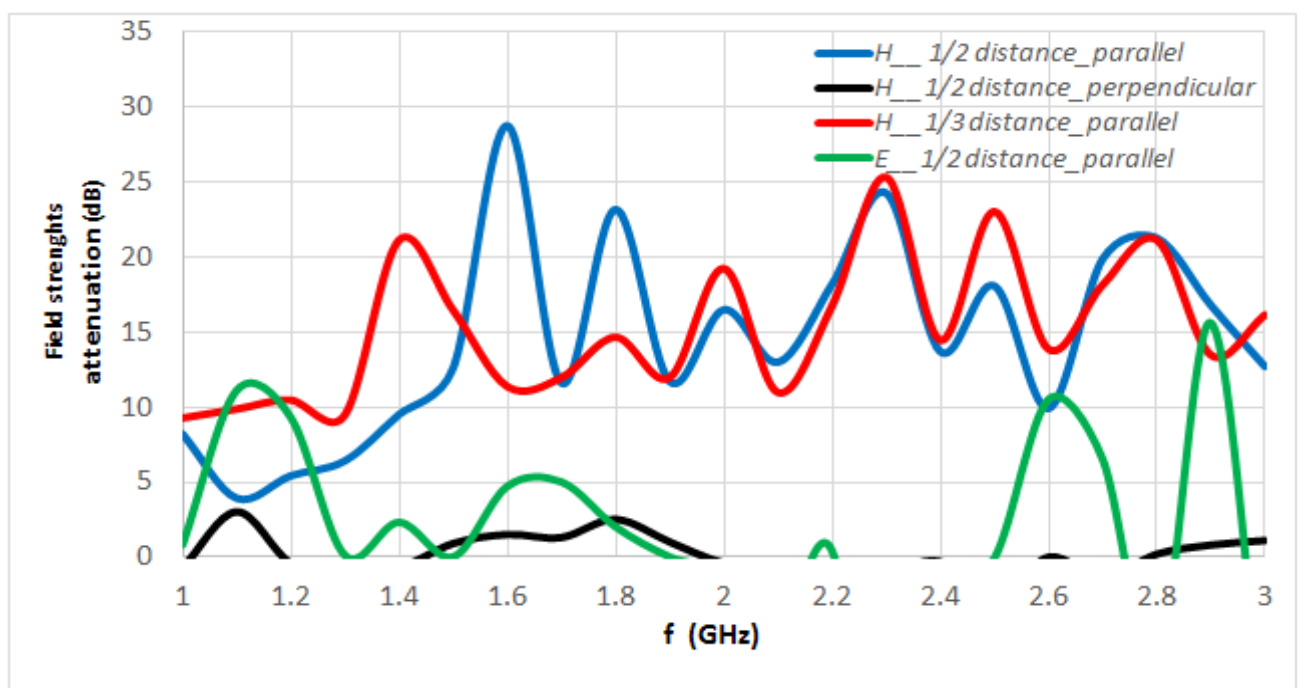

Figure no. 5 Attenuation of electromagnetic field components in the considered frequency range, for two directions of the amorphous wires and for two positions of the shield relative to the field probe. 
Figure no. 6 traces the variation of the attenuation coefficient of the $\mathrm{H}$-field strength after penetrating the fabric, in the case of progressive increasing of the incident field strength. The abscissa in Figure no. 6 shows in fact the input power fed into the emitting antenna. It is observed that up to $33 \mathrm{~W}$ of $\mathrm{P}$ in into the hyperlog antenna, the ferromagnetic wires present neither a measurable magneto-impedance effect, nor ferromagnetic resonance at any of the selected frequencies in the graphs. One must know that the used input powers conducted to incident $\mathrm{H}$-field strengths in air in the range (90-206) $\mathrm{nT}$, depending on the frequency. So, it is shown that this type of material hasn't good sensor properties at tens of nT magnetic field and the wires feature reduced sensitivities in this frequency range.

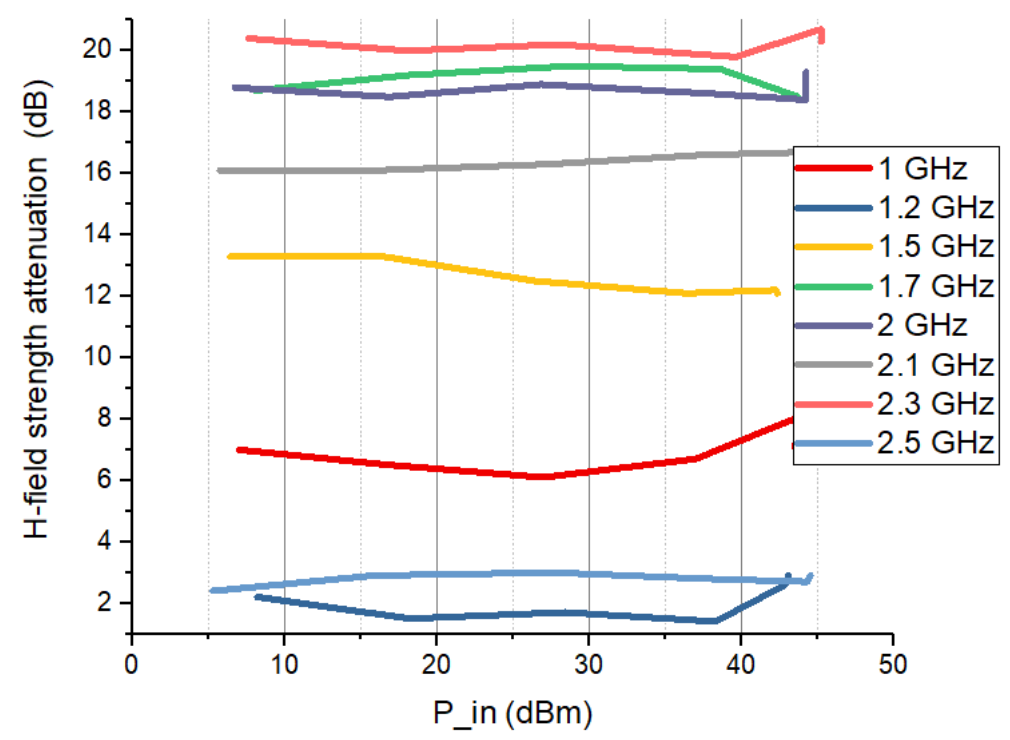

Figure no. 6 Attenuation of the H-field strength behind the fabric in function of the input power into the emitting antenna, at different frequnecies

Uncertainty budget calculation resulted in an expanded standard uncertainty (with confidence interval of $95 \%$ ) of $30 \%$.

\section{Conclusion}

By measurements of very local and separate electric and magnetic field components, we assessed the attenuation factor / shielding efficiency in the (1-3) $\mathrm{GHz}$ range of a sheet of fabric containing amorphous glass-coated ferromagnetic microwires based on $\mathrm{Co}, \mathrm{Fe}, \mathrm{Ni}, \mathrm{Mn}, \mathrm{B}$ and $\mathrm{Si}$. By means of scanning electron microscopy and energy-dispersive X-ray spectroscopy, we first characterized the structure, dimensions and chemical elements present in the fabric sample. The electromagnetic shielding properties investigations, made for the first time by using small field probes (nonstandardized methodology), showed that the component that suffers the significant attenuation is the magnetic field. The magnetic shielding coefficient is situated in the (10-25) $\mathrm{dB}$ range, with resonances at different frequencies. The shielding property of the sample is dependent on the direction of the incident field vectors relative to the amorphous wire direction. Up to incident magnetic flux densities of $200 \mathrm{nT}$, this material did not show shielding efficiency changes due to possible and expected magneto-impedance effect or ferromagnetic resonance. 


\section{REFERENCES}

Baltag, O., Cantore, C., Costandache, D., Haras, S., Larin, V., \& Torcunov, V. (2000). Study of a texture of an amorphous magnetic material able to screen non-ionizing radiation, in Di Barba, P., \& Savini, A. (eds.), Non-linear Electromagnetic Systems, IOS Press, 717-720.

Baranov, S.A., Larin, V.S., \& Torcunov, A.V. (2017). Technology, Preparation and Properties of the Cast Glass-Coated Magnetic Microwires. Crystals 7, 136.

Devkota, J., Colosimo, P., Chen, A., Larin, V. S., Srikanth, H., \& Phan, M. H. (2014). Tailoring magnetic and microwave absorption properties of glass-coated soft ferromagnetic amorphous microwires for microwave energy sensing. Journal of Applied Physics 115, 17A525.

Liberal, I. (2013). Amorphous Glass-Coated Ferromagnetic Wires in Microwave Engineering, Ph.D. Thesis. Department of Electrical and Electronic Engineering, Public University of Navarra, Department of Electrical and Electronic Engineering, Pamplona, Spain.

Makhnovskiy, D. P., \& Panina, L. V. (2006). Experimental demonstration of tunable scattering spectra at microwave frequencies in composite media containing $\mathrm{CoFeCrSiB}$ glasscoated amorphous ferromagnetic wires and comparison with theory. Physical Review B, Condensed matter 74(6).

Miclaus, S., Mihai, G., Aron, A.M., Mitrescu, C., Bechet, P., \& Baltag, O. (2017). Shielding efficiency of a fabric based on amorphous glass-covered magnetic microwires to radiation emitted by a mobile phone in $2 \mathrm{G}$ and $3 \mathrm{G}$ communication technologies. Land Forces Academy Review, vol. XXII, nr. 4 (88), 289-297.

Rau, M., Iftemie, A., Baltag, O., \& Costandache, D. (2011). The Study of the Electromagnetic Shielding Properties of a Textile Material with Amorphous Microwire. Advances in Electrical and Computer Engineering, 11(1), 17-22.

Yong-jiang, D., Jian-Jun, J., Gang, D., Bin, T., Shao-Wei, B., \& Hua-Hui, H. (2007). Magnetic and microwave properties of glass-coated amorphous ferromagnetic microwires. Transaction on Nonferrous Metal Society of China, 17, 1352-1357. 